\title{
The effect of electron pressure on suspended helium films
}

\author{
Annemarie Valkering ${ }^{1}$, Jürgen Klier, Paul Leiderer \\ Universitüt Konstanz, Fakultät für Physik, 78,457 Konstunz, Germany
}

\begin{abstract}
(Quasi) one- and zero-dimensional electron systems can be created using a suspended belium film on a structured substrate. To investigate the relation between the curved surface and the properties of the electron system, the suspended helium film profile in a quasi one-dimensional channel is measured interferometrically. It is shown that the film thickness decreases when the surface is charged with electrons at a density $>10^{8} \mathrm{~cm}^{-2}$.
\end{abstract}

Keywords; Suspended helium films; 2D electron gas; Electron pressure

It is well-known that a helium surface charged with a high number of electrons is distorted due to the electron pressure. For a bulk surface it was shown that at an electron density $n=2.4 \times 10^{9} \mathrm{~cm}^{-2}$ the surface becomes unstable dive to softening of the ripplon modes $[1,2]$. On a thin helium film, which is stabilized through van der Waals forces, this instability does not occur, but the film thickness is considerably reduced [3].

Besides bulk helium and a thin helium film, a third possible surface for electrons is a suspended helium film on a structured substrate [4]. It has the advantage that it can be used to laterally confine the clectrons on a (sub)-micrometer scale and thus create (quasi) one- and zero-dimensional electron systems. The effect of electron pressure for this system is twofold: On one hand the instability can occur, but at higher $n$ due to the larger minimum ripplon wave vector [4]. On the other hand

\footnotetext{
1 Corresponding author. E-mail: Annemarie.Valkering@uni-konstanz.de
}

the electron pressure reduces the thickness of the (curved) suspended film which eventually leads to a thin van der Waals film on the substrate in the center. This again stabilizes the film.

It is therefore of interest to investigate the helium film profile as a function of the number of electrons on the surface. We present here interferometric measurements of the ${ }^{4} \mathrm{He}$ film profile in a (single) quasi one-dimensional channel [5].

The experimental cell is described in detail in [6]. The main part consists of a substrate with a $25 \mu \mathrm{m}$ high structure with the suspended film and the electron channel in it (see inset of Fig. 1). The substrate forms one of the mirrors of the interferometer and the structure forms the electrodes for determining the transport properties of the electrons. The width of the channel is $300 \mu \mathrm{m}$. A bottom electrode below the structure provides the holding electric field for the electrons.

Fig. 1 shows the helium film profile across the channel (cross section as indicated in the inset)

Konstanzer Online-Publikations-System (KOPS)

URL: http://www.ub.uni-konstanz.de/kops/volltexte/2007/2869/

URN: http://nbn-resolving.de/urn:nbn:de:bsz:352-opus-28691 


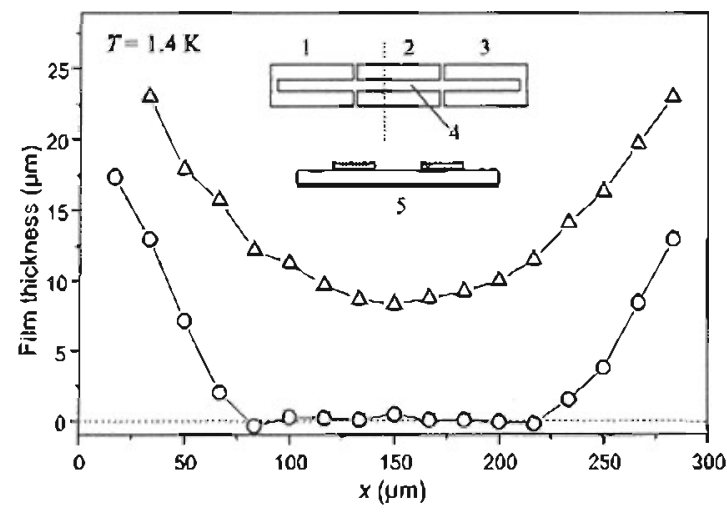

Fig. 1. Helium film profile across the channel without electrons $(\Delta)$ and with an electron density $n_{s \alpha t}=1 \times 10^{10}$ $\mathrm{cm}^{-2}(0) . n_{s a t}$ is calculated from the holding electric field, assuming saturation. Inset: Top view and cross section of the electrode structure with $1,2,3$ : electrodes, 4 : region with suspended film and electrons, 5: bottom electrode.

with and without electrons on the surface. When no electrons are present the radius of curvature $R_{c}$ of the profile can be calculated with $R_{c}=\alpha / \rho g H$, with $\alpha$ the surface tension of liquid helium and $H$ the bulk level below the structure [ $[$ ].

With $n_{\text {sat }}=1 \times 10^{10} \mathrm{~cm}^{-2}$ electrons on the surface (saturated density calculated from the holding field) the profile lias considerably changed. The bulk helium level is monitored during the measurement with a level capacitor and does not change within our experimental resolution $(\approx 5 \mu \mathrm{mi})$. A helium film thickness change of $10 \mu \mathrm{m}$ in the center requires a bulk helium level change of $0.2 \mathrm{~mm}$. We can therefore conclude that the change in helium film thickness is not due to an -inadvertentchange in bulk helium level.

In the center of the channel over a width of $150 \mu \mathrm{m}$ the film has become flat. We assume that we have a thin van der Waals film on the substrate, whose thickness, however, cannot be resolved with our method. The effect of electron pressure on the local $R_{c}$ of the suspended film is given by: $R_{c}=$ $\alpha /\left(\rho g H+\frac{n^{2} e^{2}}{2 \varepsilon_{0} \varepsilon_{\mathrm{Hc}}}\right)$. From this equation it follows that an electron density of $1 \times 10^{9} \mathrm{~cm}^{-2}$ is needed to reduce the film thickness in the center from initially $9 \mu$ m to zero (i.e. van der Waals film). We ubserved this reduction for $n_{\text {sat }}>2.5 \times 10^{9} \mathrm{~cm}^{-2}$ (data not shown). The discrepancy between these two values can be explained if we have a nonsaturated electron sheet on the surface.

Another indication of the number of elestrons on the surface can be obtained from the transport properties of the electrons in the channel. The capacitive coupling of the electrons to the electrodes was measured as a function of temperature for the electron sheet with $n_{\text {sat }}=1 \times 10^{10} \mathrm{~cm}^{-2}$. A sharp kink in the amplitude was observed at $T=850 \mathrm{mK}$, whicli we interpreted as a phase transition to the (2D) Wigner crystal. Since the crystallization temperature is density dependent, $n$, can be obtained in this way. The density corresponding to $850 \mathrm{mK}$ is 1.5 to $2.3 \times 10^{9} \mathrm{~cm}^{-2}$, where the screening of the glass substrate is taken into account in the second value. This screening is important for the thin film region. The measured $n$ is again lower than the saturated one. The ratio $n / n_{\text {sat }}$ should be constant. This is reasonable because the surface is charged continuously while the holding electric field is ramped to its final value. So we conclude that the decrease in film thiokness is in agreement with theory. Since the electron density profile falls off rather sharply towards the edge [7] we can also conclude that the electrons in the center contribute to the capacitive coupling.

We thank R. v.d. Heijden for discussions. This work was supported by the A. v. Humboldt Fonndation and the 'Forschergruppe Quantengase'.

\section{References}

(1] A.P. Volodin, M.S. Khaikin, V.S. Edel'man, JETP Lett 26 (1977) 543

[2] M. Wanner, P. Leiderer, Phys. Rev. Lett. 42 (1979) 315

[3] H. Etz, W. Gomber1, W. Idstein, P. Leiderer, Phys Rev. Lett. 53 (1984) 2567.

[4] D. Marty, J. Phys. C: Solid State Phys. 19 (1986) 6097

[5] A.M.C. Valkering, P.K.H. Sommerfeld, P.J. Richardson, R.W. wan der Heijden, A.T.A.M. de Wrele, Czech. 3. Phys. 46 (1996) 321.

[6] A.M.C. Vilkering, J. Klier, E. Teske, R.W. van der Heijden, P. Leiderer, J. Low Temp. Phys. 113 (1998) 111.5 .

(7) A.M.C. Valkering, R.W, van der Heijden, Physica 249251 B (1998) 652 